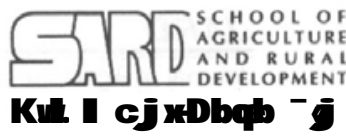

Available online at http: / / www.banglajol.info/index.php/jard
J ARD

Journal of Agriculture \& Rural Development

\title{
Genetic Divergence in Pointed Gourd
}

\author{
M. Y. KABIR ${ }^{1}$, A. S. M. M. R. KHAN ${ }^{2 *} \&$ M. S. HASSAIN ${ }^{3}$ \\ ${ }^{1}$ National Bank, Dhaka, Bangladesh \\ ${ }^{2}$ OFRD, Bangladesh Agricultural Research Institute, Gazipur, Bangladesh \\ ${ }^{3}$ Regional Agricultural Research Station, Ishurdi, Pabna, Bangladesh
}

\begin{abstract}
The experiment was conducted at the Regional Agricultural Research Station, Ishurdi, Pabna during the growing season 2005-2006 to estimate the genetic diversity among 24 genotypes of pointed gourd by using Mahalanobis $D^{2}$ statistics for nine characters. The genotypes were grouped in to five clusters. The cluster I and III consisted of highest number of genotypes and it was six. The cluster IV contained the lowest number of genotypes and it was three. The clustering pattern of the genotypes under this study revealed that the genotypes collected from the same location were grouped into different clusters. The genotypes of Kushtia were distributed in different clusters. The inter cluster distance were larger than the intra cluster distance suggesting wider genetic diversity among the genotypes of different groups. The highest intra cluster distance was computed for cluster IV (35.80) and the minimum intra cluster distance was found in cluster III (18.37). The clusters IV and II were more diverse as indicated by maximum inter cluster distances between them (41.56) and the minimum inter cluster divergence was observed between cluster III and II (6.84). Cluster II had the highest cluster mean value for number of fruits per plant (391), weight of fruit per plant (11.72kg) and yield (35.28t/ha). Genotypes of the cluster $V$ had late maturity.
\end{abstract}

Key words: Genotype, genetic diversity, pointed gourd.

\section{INTRODUCTION}

Pointed gourd (Trichosanthes dioica Roxb) is an economically important cucurbit and is extensively propagated through vegetative means, viz vine and root cuttings. Because of continued vegetative propagation and judicious selection, considerable variability with respect to fruit number of cultivars and forms having are available. In crop improvement programme genetic diversity has been considered as an important factor which is also essential pre-requisite/tool for hybridization programme to obtain high yielding progenies. For planning and executing genetic improvement programme a clear understanding of the magnitudes of genetic diversities for yield and its component characteristics are important to plant breeders for both cross and self quantification of genetic diversity. The present study was therefore undertaken to analyze the genetic divergence of pointed gourd.

* Corresponding author: Senior Scientific Officer (OFRD), Bangladesh Agricultural Research Institute, Gazipur, Bangladesh

(C) 2009 School of Agriculture and Rural Development, Bangladesh Open University, All rights reserved. 


\section{MATERIALS AND Methods}

The investigation was carried out at the Regional Agricultural Research Station, Ishurdi, Pabna during the growing season 2005-2006. The experiment was laid out in RCB design with three replications. Twenty four pointed gourd accession were collected from north western region. Each pointed gourd accession was considered as individual treatment of the experiment. Therefore, there were 24 treatments of the experiment. Unit plot size was $1.25 \mathrm{~m} \times 4.0 \mathrm{~m}$ and plant spacing was $1 \mathrm{~m} \times 1.25 \mathrm{~m}$. The crop was grown following the recommended cultural practices (Rashid, 1999). Nine characters of 24 genotypes of pointed gourd related to plant, flower, fruit, seed and yield were used for genetic diversity analysis. These nine characters were days to $1^{\text {st }}$ flowering, number of node at first harvest, fruit length, fruit width, fruit weight, pulp seed ratio, number of fruits per plant, weight of fruits per plant and yield. Genetic diversity was studied following Mahalanobis (1936) generalized distance $\left(D^{2}\right)$ extended by Rao (1952). Tochers method (Rao 1952) was followed for determining the group constellations. Canonical analysis was also done according to Rao (1964) to confirm the results of clusters and $D^{2}$ analysis. Statistical analysis was carried out using IBM computer.

\section{Results And Discussion}

Twenty four pointed gourd accessions grouped into five different clusters (Table 1). Clusters I and III contained the highest number of accessions (6) followed by cluster V (5) and cluster II (4), respectively. The lowest number of accessions (3) was found in the clusters IV. The clustering pattern of the accessions under this study revealed that the accessions collected from the same location were grouped into different clusters. Masud et al. (1995) reported similar results in sweet gourd, Mannan et al. (1993) in pani kachu, Singh and Singh (1979) in okra and Ram (2001) and Khan (2006) in pointed gourd.

Table 1. Distribution of 24 pointed gourd accessions in $\mathbf{5}$ clusters

\begin{tabular}{ccll}
\hline Cluster no. & $\begin{array}{c}\text { Total no. of accessions } \\
\text { in cluster }\end{array}$ & $\begin{array}{c}\text { Accessions included in different } \\
\text { clusters }\end{array}$ & Source \\
\hline I & 6 & $\begin{array}{l}\text { PG002,PG003 and PG004 } \\
\text { PG014,PG015 and PG018 }\end{array}$ & $\begin{array}{l}\text { Pabna } \\
\text { Kushtia }\end{array}$ \\
II & 4 & $\begin{array}{l}\text { PG007 and PG012 } \\
\text { Kushtia }\end{array}$ \\
III & 6 & PG023 and PG024 & Bogra \\
& & PG005,PG008 and PG009 & Kushtia \\
IV & 3 & PG016,PG017 and PG020 & Gaibandha \\
V & 5 & PG001,PG006 and PG022 & Jessore \\
& & PG021 and PG011 & Rangpur \\
& & & Maherpur \\
\hline
\end{tabular}

The intra and inter cluster distance $\left(D^{2}\right)$ are presented in Table 2. Statistical distances represent the index of genetic diversity among the clusters. The inter cluster distances were larger than the intra cluster distances suggesting wider genetic diversity among the genotypes of different groups (Table 2). Uddin and Mitra (1994) and Khan (2006) obtained higher inter cluster distances than the intra cluster distances in multivariate analysis in sesame and pointed gourd respectively. Based on the nine quantitative characters of pointed gourd genotypes, the intra cluster distance was computed by using the values of inter genotypes distance from distance matrix according to Singh and Chaudhuary, (1985). The highest intra cluster distance was computed for cluster IV (35.80) followed by cluster I (28.12) and cluster $\vee(26.63)$, respectively. The minimum intra cluster distance was found in cluster III (18.37). Somayagullu et al. (1970) reported that the clustering relatively instability due to relatively lesser divergence, where as widely diverged cluster remained distinct in different environment. So the cluster IV, I and V would be more stable. The lines belonging to distinct characters could be used in hybridization program for obtaining a wide spectrum of variation among 
segregates. The clusters IV and II were more diverse as indicated by maximum inter cluster distances between them (41.56) followed by the distance among clusters IV and III (36.35), II and I (27.41), V and IV (24.15), III and I (21.44), V and II (19.44), IV and I (16.15) and between V and III (13.25). The maximum values of inter cluster distance indicated that the accessions belonging to cluster IV were far away from those of cluster II. The minimum inter cluster divergence was observed between cluster III and II (6.84) indicating that the genotype of these cluster were genetically closed. Similar results were also found by Prasad (1995) in bush bean and Khan (2006) in pointed gourd. Higher inter and intra- cluster distance indicates higher genetic variability among accessions between and within clusters, respectively. The minimum inter and intra-cluster distance indicates closeness among the accessions of two clusters and within the cluster also.

Accessions among the clusters separated by high $D^{2}$ values could be used in hybridization program for obtaining wide spectrum of variations among the segregates (Bhatt, 1970 and Seetharaman et al.1988). It is revealed that crosses should be made between accessions belonging to the distant clusters for high heterotic response. In the present study, the inter cluster distances between clusters ranged from 6.84 to 41.56 suggesting crossing accessions of cluster IV with desirable genotypes of others clusters would express heterosis. Mian and Bhal (1989) reported that parental clusters separated by medium $\mathrm{D}^{2}$ values had significant positive heterosis. Thus, heterosis could also be exploited by crossing between accessions belonging to clusters with moderate diversity like between accessions of cluster II \& IV, cluster III \& IV and IV\& V is likely to produce new recombinant with desired traits.

Table 2. Average inter and intra-cluster distance $\left(D^{2}\right)$ for $\mathbf{2 4}$ pointed gourd accessions

\begin{tabular}{cccccc}
\hline Cluster & I & II & III & IV & V \\
\hline I & 28.12 & 27.41 & 21.44 & 16.15 & 8.72 \\
II & & 23.53 & 6.84 & 41.56 & 19.44 \\
III & & & 18.87 & 36.35 & 13.25 \\
IV & & & 35.80 & 24.15 \\
V & & & & 26.63 \\
\hline
\end{tabular}

The cluster means of different characters of 24 accessions of pointed gourd are presented in Table 3. Cluster I was composed of six accessions. None of the 9 characters had the highest mean value but lowest fruit length $(9.93 \mathrm{~cm})$ was found in cluster I. Cluster II comprising four accessions, the mean values of cluster II ranked first for number of fruits per plant (391), weight of fruit per plant (11.72kg) and yield (35.28t/ha). Prasad et al. (1993) also reported similar findings in cucumber. Cluster III comprising six accessions had the highest cluster mean value for days to first flowering (89 day) and fruit weight (48.7g). Cluster IV was composed of three accessions and the highest mean value was found in fruit length $(10.40 \mathrm{~cm})$ and pulp seed ratio (14.48). The lowest number of fruits per plant (48) was found in IV. Cluster $V$ was composed of 5 accessions and had the highest cluster mean value for number of node at first harvest (39.4) and fruit weight (46.97g). Accessions of the cluster $\mathrm{V}$ had early maturity.

Table 3. Cluster mean values for yield and yield contributing characters of pointed gourd

\begin{tabular}{lccccc}
\hline Characters & I & II & III & IV & V \\
\hline Days to $1^{\text {st }}$ flowering (day) & 88.45 & 87.50 & 89.00 & 82.33 & 81.53 \\
Number of node at first harvest & 33.11 & 35.58 & 35.22 & 25.33 & 39.40 \\
Fruit length $(\mathrm{cm})$ & 9.93 & 10.00 & 10.33 & 10.40 & 10.05 \\
Fruit breadth $(\mathrm{cm})$ & 4.27 & 3.75 & 3.59 & 3.46 & 3.63 \\
Single fruit weight(g) & 35.91 & 44.50 & 47.69 & 36.09 & 46.97 \\
Pulp seed ratio & 13.49 & 11.70 & 13.61 & 14.48 & 12.97 \\
Number of fruits per plant & 152 & 391 & 335 & 48 & 240 \\
Weight of fruit per plant $(\mathrm{kg})$ & 5.88 & 11.72 & 11.34 & 1.45 & 8.32 \\
Yield (t/ha) & 17.64 & 35.28 & 33.98 & 12.46 & 24.99 \\
\hline
\end{tabular}




\section{Contribution of different characters towards divergence}

The characters contributing more to the divergence are given greater emphasis for deciding on the cluster for the purpose of further selection and the choice of patterns for hybridization (Jagadev et al., 1991). The results of Canonical vector analyses revealed that in vector I (Z1), the important characters responsible for genetic divergence in the major axis of differentiation were days to first flowering, fruit length and pulp seed ratio (Table 4). In vector II (Z2), days to first flowering, number of node at first harvest, fruit breadth, number of fruit per plant, weight of fruit per plant and yield played a major role in the second axis of differentiation. The role of days to first flowering in both the vectors indicated the important component of genetic divergence among the 24 pointed gourd accessions. Negative values in both the vectors for fruit weight indicated that the lowest contribution to the total divergence. Hence, considerable emphasis should be given on these characters to increase fruit yield in pointed gourd. Mathew et al. (1986) reported that fruit weight per plant was the major contributor towards divergence in Cucumis melo. Masud et al. (1995) found that fruit weight was one of the important contributors to genetic divergence in sweet gourd. Khan (2006) observed that fruit weight, number of fruits per plant and weight of fruits per plant were the higher contributors to the divergence in pointed gourd. Based on $D^{2}$ analysis, Anitha and Dorairaj (1990) grouped 8 parents and 56 hybrids into 15 clusters and concluded that the days to flower was the important contributors to the genetic divergence.

Table 4. Relative contribution of different characters towards divergence

\begin{tabular}{lcc}
\hline \multicolumn{1}{c}{ Characters } & Vector I & Vector II \\
\hline Days to $1^{\text {st }}$ flowering & 0.0136 & 0.2937 \\
Number of node at first harvest & -0.3567 & 0.0609 \\
Fruit length $(\mathrm{cm})$ & 0.351 & -0.4584 \\
Fruit breadth (cm) & -0.0364 & 0.5612 \\
Single fruit weight & -0.399 & -0.4062 \\
Pulp seed ratio & 0.1145 & -0.4484 \\
Number of fruits per plant & -0.4742 & 0.0267 \\
Weight of fruits per plant & -0.4925 & 0.1344 \\
Yield of ton per hectare & -0.5010 & 0.01224 \\
\hline
\end{tabular}

\section{Selection of accessions for future improvement}

The crosses involving parents belonging to the maximum divergent clusters were expected to manifest maximum heterosis and also wide variability in genetic architecture. Ramanujam et al. (1974) in mungbean and Mian and Bhal (1989) in chickpea reported that parental clusters separated by medium $D^{2}$ values exhibited significant and positive heterosis for seed yield and some of its components in mungbean. Similar findings were also observed by Masud et al. (1995) in sweet gourd and Khan et al. (1995) in pointed gourd. Thus cross between the genotypes of cluster $\mathrm{V}$ with those of cluster II and III would exhibit heterosis and is likely to produce new recombinants with desired characters in pointed gourd.

Considering the magnitude of genetic distance, contribution of different characters towards the total divergence and magnitude of cluster means for different characters performance, the following genotypes were considered to perform better if used in hybridization program.

The accessions PG001 of cluster $V$ could be selected for earliness and fruit weight. The accessions PG008, PG009 and PG013 of the cluster III could be selected for number of fruits per plant, higher fruit weight per plant and yield. The accessions PG016, PG019 and PG022 of the cluster IV could 
be selected for fruit length. The accessions PG007, PG012 and PG023 of the cluster II could be selected for breadth of fruit. From cluster I the accessions PG003, PG004 and PG014 could be selected for higher pulp seed ratio.

\section{Literature Cited}

Anitha, N. and M. S. Dorairaj, M. S. 1990. Genetic divergence and hybrid performance in sesame. J. Oilseeds Res., 7(1), 63-71.

Bhatt, G. M. 1970. Multivariate analysis approach to selection of parents for hybridization aiming at yield improvement in self-pollinated crops. Aust. J. Agric. Res., 20, 1-7.

Gomez, K. A. and A. A. Gomez. 1984. Statistical Procedure for Agricultural Research. John Wiley and Sons. Inc. New York. pp. 67-215.

Hazra, P.; R. Ghosh; Nath-Subhadeep and S. Nath. 2003. Identification of important yield components in pointed gourd (Tricosanthes dioicaRoxb.). Crop Res. Hisar, 25(2), 244-252.

Jain, S. K.; C. O. Qualest.; G. M. Bhatt and K. K. Wu. 1975. Geographical patterns of phenotypic diversity in a world collection of duram wheats. Crop Sci., 15, 700-704.

Jagadev, P. N.; K. M. Samal, and L. Lenka. 1991. Genetic divergence in rape mustard. Indian J. Genet., 51, 465-466.

Khan, A.S.M.M.R. 2006. Study of Genetic Diversity and Production Technology of Pointer gourd..Ph.D. Thesis, Bangladesh Agricultural Univ., Mymensingh, Bangladesh. pp. 77-93.

Mahalanobis, P. C. 1936. On the generalized distance in statistics. Proc. Nat. Inst. Sci. Ind., 2, 4955.

Mathew, S. M.; P. K. Gopalakrishan and K. V. Peter. 1986. Genetic distance among five botanical varieties of Cucumis melo. Agric. Res. J. Kerala. 24(2), 195-196.

Mannan, M. A.; M. S. Ahmad; M. M. Rashid; M. K. R. Bhuiyan and R. Gomes. 1993. Genetic diversity of Colocasia esculenta (L.) Schtt. Root Crops, 19(2), 95-99.

Masud, M. A. T.; M. A. Z. Chowdhury; M. A. Hossain and S. M. M. Hossain. 1995. Multivariate analysis in pumpkin (Cucurbita moschata Duch ex Poir). Bangladesh J. Plant Breed. Genet., 8(1\&2), 45-50.

Mian, M. A. K. and P. N. Bhal. 1989. Genetic divergence and hybrid performance in chickpea. Indian J. Genet., 49(1), 119-129.

Prasad, V. S. R. K., D. P. Singh and R. P. Singh.1993. Biological divergence in the land races of Indian cucumber (Cucumis sativus L.). Indian J. Hort., 50(1), 57-63.

Prasad, V. S. R.; B. P. Jain.; S. P. P. Verma and D. K. Ganguly. 2001. Diversity pattern and choice of parents of hybridization in slicing cucumber (Cucumis sativus L.). J. Res. Bires. Agril. Univ., 13(5), 33-39.

Prasad, V.S.R.K. 1995. Genetic divergence of yield and its component in bush bean. Indian J. Hort., 527(1), 146-150.

Ram, D. 2001. Non- hierarchical Euclidean cluster analysis in pointed gourd. Indian J. Hort., 58(3), 264-268.

Ramanujam, S.; A. S. Tiwary and R. B. Mehra. 1974. Genetic divergence and hybrid performance in mungbean, Theor. Appl. Genet., 44(5), 211-214.

Rao, C. R. 1952. Advanced Statistical Methods in Biometrical Research. John Wiley and Sons, New York. pp. 45-110. 
M. Y. Kabir et al.

Rao, C. R. 1964. The use and interception of Principle Component analysis in Applied Research. Sankhya. 22, 317-318.

Rashid, M. M. 1999. Vegetable Science (in Bangla). $1^{\text {st }}$ ed., Bangla Academy, Dhaka. Bangladesh. pp. 333-336.

Seetharaman, R.; N. De.; R. M. K. Sinha and S. P. Banerjee. 1988. Genetic divergence in rice. Indian. J. Genet., 48, 189-194.

Singh, R. K. and B. D. Chaudhury. 1985. Biometrical methods of quantitative genetic analysis. Harayana J. Hort. Sci., 12(1), 151-156.

Singh, S. P. and H. N. Singh. 1979. Genetic divergence in okra (Abelmoschus esculentus L. Moerch). Indian J. Hort., 36(2), 166-170

Somayagullu,P.N. ; A.B Joshi and B.R. Murty. 1970. Genetic divergence in wheat. Indian J. Genet. Plant Breeding. 30, 47-58.

Uddin, M. J. and B. N. Mitra. 1994. Variability of some quantitative characters in sesame (Sesamum indicum L.). Bangladesh J. Agric. Sci., 19, 17-22. 